Математички Билтен

ISSN 0351-336X (print)

41(LXVII) No. 2

ISSN 1857-9914 (online)

2017(54-73)

UDC: $519.876 .5-75$

Скопје, Македонија

\title{
MATHEMATICALLY UNIVERSALIZED ESTIMATIONS OF MEDICAL RESULTS
}

\author{
NENAD O. VESIĆ, LANA MAČUKANOVIĆ-GOLUBOVIĆ, AND DUŠICA ILIĆ
}

\begin{abstract}
The methodology of medical-data-analysis is improved here. Three novel computational algorithms for medical data analysis are presented in this paper. The first of them analyzes a state of single patient. The second algorithm analyzes a status of group of patients based on states of single patients from this group. The third algorithm compares previously analyzed effects of different groups of patients. Measure characteristics of results of the first algorithm are examined.
\end{abstract}

\section{INTRODUCTION}

Medical scales have an important role in medical-data-analysis. These scales help doctors in decision-making about therapies. Different diseases are covered by different corresponding scales (see for example [7]-FSS and EDSS and [18]-Glasgow coma scale and FOUR scale). Previous mentioned scales, such as many other ones, are different and they stay focused on specific disabilities of states of single patients. However, state-estimates obtained from different scales cannot be combined.

Humans, animals, insects, etc. are not structured of independent cells, organs, tissues and organic systems. All parts of live beings are connected and any disorder in one part disables other parts of bodies to operate. In this paper, we will use different mathematical knowledge (the measure theory $[3,5,8,9,11-13,15]$, the theory of statistics $[1,2]$ and differential geometry $[16,17])$, to explain what "sizes" of medical results mean. In this way, we will help doctors to understand real details about disordered health-states.

1.1. On the measure theory. The most applicable mathematical theory is the measure theory together with the corresponding generalizations.

2010 Mathematics Subject Classification. Primary: 97M60, 28E05 Secondary: 47A56, 28A10, $26 \mathrm{E} 35$.

Key words and phrases. algorithm, measure, pseudo-additive measure, matrix, curve, expectation, signed area, medication effect, estimate, graphics. 
Definition 1.1: [11] Let $\Omega$ be a set and $\Sigma$ be a $\sigma$-algebra over $\Omega$. A function $\mu: \Sigma \rightarrow \mathbb{C}$ which satisfies the properties

1. $\mu(\emptyset)=0$

2. $\mu\left(\cup_{n=1}^{+\infty} A_{n}\right)=\sum_{n=1}^{+\infty} \mu\left(A_{n}\right)$, for disjoint sets $A_{n} \in \Sigma, n=1,2, \ldots$

is said to be the ( $\sigma$-additive) complex measure.

Remark 1: A real function $\nu: \Sigma \rightarrow \mathbb{R} \cup\{+\infty\}$ is the $\sigma$-additive real measure if it, together with the conditions 1 . and 2 . from the previous definition, satisfies the condition $\nu(A) \geq 0$ for all $A \in \Sigma$.

The $\sigma$-additive measure theory is generalized. For this generalization, it was necessary to be defined the term of pseudo-addition.

Definition 1.2: [15] A binary operation $*$ on $\mathbb{C}^{2}$ which satisfies the followings:

(P1) $x * 0=0 * x=x$,

(P2) $(x * y) * z=x *(y * z)$,

(P3) If $x \leq x^{\prime}$ and $y \leq y^{\prime}$ then $x * y \leq x^{\prime} * y^{\prime}$,

(P4) If $x_{n} \underset{n \rightarrow+\infty}{\longrightarrow} x$ and $y_{n} \underset{n \rightarrow+\infty}{\longrightarrow} y$ then $x_{n} * y_{n} \underset{n \rightarrow+\infty}{\longrightarrow} x * y$.

is the pseudo-addition.

Definition 1.3: [15] A set function $\mu$ defined on a $\sigma$-algebra $\Sigma$ is said to be the pseudo-additive measure (with regard to pseudo-addition $*$ ) if it satisfies the following conditions:

1. $\mu(\emptyset)=0$,

2. $\mu\left(A_{1} \cup A_{2} \cup \ldots\right)=\mu\left(A_{1}\right) * \mu\left(A_{2}\right) * \ldots$, for disjoint sets $A_{n} \in \Sigma$,

3. If $A_{1} \subseteq A_{2} \subseteq \ldots$ and $A_{n} \underset{n \rightarrow+\infty}{\longrightarrow} A \in \Sigma$ then $\mu\left(A_{n}\right) \underset{n \rightarrow+\infty}{\longrightarrow} \mu(A)$, monotonously.

Statistics is one of the most useful measures into human lives, [2]. However, D. Bowers [1] warns doctors that their unconditional believing to results of statistical data-analysis may be very dangerous.

Let $X$ be a random variable which takes values $x^{1}, \ldots, x^{n}$ with the corresponding probabilities $p_{1}, \ldots, p_{n}$. Expectation $E X$ of the variable $X$ is

$$
E X=p_{1} x^{1}+\ldots p_{n} x^{n} .
$$

Specially, in the case of $p_{1}=\ldots=p_{n} \quad(=1 / n)$ the expectation $E X$ is

$$
E X=\frac{1}{n}\left(x^{1}+\ldots+x^{n}\right) .
$$

1.2. Coordinate matrix curves. A curve $\ell$ in the $N$-dimensional Descartes space $\mathbb{R}^{N}$ is the vector function $\ell:(a, b) \rightarrow \mathbb{R}^{N}$. The term of curve is generalized in [16].

Definition 1.4: A matrix valued function

$$
M=M(t)=\left[\begin{array}{ccc}
m_{1}^{1}(t) & \ldots & m_{q}^{1}(t) \\
\vdots & \ddots & \vdots \\
m_{1}^{p}(t) & \ldots & m_{q}^{p}(t)
\end{array}\right]
$$


for $m_{v}^{u}:\left[a_{v}, b_{v}\right] \rightarrow \mathbb{C}, u=1, \ldots, p, v=1, \ldots, q$, is said to be the coordinate matrix curve (of the type $p \times q$ ) on the set $\mathbb{D}=\left[a_{1}, b_{1}\right] \times \ldots \times\left[a_{q}, b_{q}\right]$.

Let be $U=\left\{u_{1}, \ldots, u_{k}\right\} \subseteq\{1, \ldots, p\}$. A coordinate matrix curve

$$
M_{U}=M_{U}(t)=\left[\begin{array}{ccc}
m_{1}^{u_{1}}(t) & \ldots & m_{q}^{u_{1}}(t) \\
\vdots & \ddots & \vdots \\
m_{1}^{u_{k}}(t) & \ldots & m_{q}^{u_{k}}(t)
\end{array}\right]
$$

is the $u_{1}-\ldots-u_{k}$-curve of the coordinate matrix curve $M$.

Coordinate matrix curves

$$
\mathcal{P}_{M}(t)=\sum_{r=1}^{p} M_{r}(t) \quad \text { and } \quad \mathcal{M}_{M}(t)=\frac{1}{p} \mathcal{P}_{M}(t)
$$

are the para-curve and the mid-para-curve of the coordinate matrix curve $M$, respectively.

Let $\mu=\mu(t)=\left[\begin{array}{lll}\mu_{1} & \ldots & \mu_{q}\end{array}\right]$ be a coordinate matrix curve of the type $1 \times q$ defined on a set $\left[t_{1}, t_{2}\right] \times\left[t_{2}, t_{3}\right] \times \ldots \times\left[t_{q}, t_{q+1}\right]$ and let be $t_{0} \in\left[t_{k}, t_{k+1}\right]$. A sum

$$
\mathcal{A}_{\mu}^{t_{1}, \ldots, t_{q+1} ; k}\left(t_{0}\right)=\sum_{r=1}^{k-1} \int_{t_{r}}^{t_{r+1}} \mu_{r}(t) d t+\int_{t_{k}}^{t_{0}} \mu_{k}(u) d u
$$

is the signed area of the matrix curve $\mu$ at the time $t_{0}$.

1.3. Motivation. As we said above, D. Bowers [1] insists on carefulness in medicaldecision-making based on conclusions obtained from statistical data-analysis. Moreover, statistics is not a daily applicable tool. A method, based on a simple computations, would be useful in daily medical-decision-makings.

There is one more problem with statistical data analysis. Based on statistical analysis, researchers obtain results as there is or there is not a significant difference between two populations. The following questions arise:

- What exactly is a significant difference?!

- Are results of two analyzed populations suitable to be compared?!

A relatively simple computational algorithm for medical-data-analysis was constructed in the year of 2002 (see [17]). Authors tried to apprise changes of states of chronically ill patients. The more general problem is how to estimate changes of states of a patient who is ill of a random disease.

The purposes of this paper are the following ones:

Purpose 1: To find a better classification of medical tests then the classification presented in [17],

Purpose 2: To perform general estimates of some results presented in [17],

Purpose 3: To approximate changes of medical results through the time. 


\section{Algorithms For DAily medical applications}

As it was mentioned above, different medical disorders are estimated with the help of the corresponding scales. Because these scales are different, it would be useful to overcome the differences between them.

2.1. Results of a single patient. Let us numerically estimate results of a patient. Similarly as in [17], medical tests are classified in five disjoint groups:

$\mathbf{G}_{1}$ Positive-negative tests:

Medical tests which results have one of the forms it is positive result (it is detected a disorder in test) or it is negative result (it is not detected any disorders in test) are classified in this group of tests. Examples of tests which are classified in this group are some subjective feelings of a patient, anti-body-tests, viral tests, etc. An estimate of result $\mathbf{r}^{(\mathbf{1})}$ of a test from this group is

$$
e^{1}\left(\mathbf{r}^{(\mathbf{1})}\right)= \begin{cases}0, & \text { the result } \mathbf{r}^{(\mathbf{1})} \text { is negative } \\ 1, & \text { the result } \mathbf{r}^{(\mathbf{1})} \text { is positive }\end{cases}
$$

$\mathbf{G}_{2}$ Positive-positive tests:

Medical tests which results have more degrees (rates) of positivity are classified in this group of tests. Results of some tests from this group are numerically rated, but some other tests from this group are descriptively explained.

As same as in [17], the least intensive disorder of a descriptively explained result is rated with 1 . If positive results of a test from this group of some special intensity are rated with $k_{0}$, minimally more intensive positive results of these ones are rated with $k_{0}+1$.

Any positive result of a test from this group is numerically rated in this way. Let all possible numerical rates of positive result $\mathbf{r}^{(\mathbf{2})}$ of a test from this group be

$$
\alpha_{1}^{(2)}<\alpha_{2}^{(2)}<\ldots<\alpha_{k}^{(2)}
$$

and let the positive result $\mathbf{r}^{(2)}$ be rated with $\alpha_{0}^{(2)} \in\left\{\alpha_{1}^{(2)}, \ldots, \alpha_{k}^{(2)}\right\}$.

There are three cases: (lipr) If rates $\alpha_{u}^{(2)}$ and $\alpha_{v}^{(2)}$ of results of this test satisfy $\alpha_{u}^{(2)}<\alpha_{v}^{(2)}$ then the disorder rated with $\alpha_{u}^{(2)}$ is less intensive than the disorder rated with $\alpha_{v}^{(2)} ;\left(\right.$ mipr) If rates $\alpha_{u}^{(2)}$ and $\alpha_{v}^{(2)}$ of results of this test satisfy $\alpha_{u}^{(2)}<\alpha_{v}^{(2)}$ then the disorder rated with $\alpha_{v}^{(2)}$ is less intensive than the disorder rated with $\alpha_{u}^{(2)}$; In the third case, which one is not lipr nor mipr, this result has to be rated descriptively and, after that, the corresponding numerical rate of this result is the rate from the case lipr.

The estimate of the result $\mathbf{r}^{(2)}$ rated with $\alpha_{0}^{(2)}$ is 
$\mathbf{G}_{3}$ Walking test:

$$
e^{2}\left(\mathbf{r}^{(2)}\right)= \begin{cases}0 & \text { negative result } \\ \frac{\alpha_{0}^{(2)}+\alpha_{k}^{(2)}-2 \alpha_{1}^{(2)}}{\alpha_{k}^{(2)}-\alpha_{1}^{(2)}}, & \text { positive result } \\ \frac{-\alpha_{0}^{(2)}-\alpha_{1}^{(2)}+2 \alpha_{k}^{(2)}}{\alpha_{k}^{(2)}-\alpha_{1}^{(2)}}, & \text { in the case of lipr } \\ & \text { positive result } \\ & \text { in the case of mipr. }\end{cases}
$$

Only one medical test is classified in this group of test. A result of this test is the distance which patient may walk continually together with the corresponding fact does this patient walk alone or he needs a help (a stick, a wheelchair, etc.). Let this distance be $\mathbf{r}^{(3)}=d$ kilometers. The estimate of this result is

$$
e^{3}\left(\mathbf{r}^{(\mathbf{3})}\right)= \begin{cases}0.1, & \text { patient walks alone more than } 1 \text { kilometer } \\ 1 / d, & \text { patient walks alone } d \text { kilometers } \\ i / d, & 0<d<1, \\ 10^{3} i, & d=0,\end{cases}
$$

for $i^{2}=-1$.

$\mathbf{G}_{4}$ Tests with two finite reference ranges:

Medical tests with negative results between special numerical borders (named the reference ranges) are classified in this group of tests. Let $\mathbf{r}^{(4)}$ be a result of test with reference ranges $\alpha^{(4)}$ and $\beta^{(4)}, \alpha^{(4)}<\beta^{(4)}$. The estimate of this result is

$$
e^{4}\left(\mathbf{r}^{(4)}\right)= \begin{cases}0, & \alpha^{(4)} \leq \mathbf{r}^{(4)} \leq \beta^{(4)}, \\ \frac{\mathbf{r}^{(4)}-\beta^{(4)}}{\beta^{(4)}-\alpha^{(4)}}, & \mathbf{r}^{(4)}>\beta^{(4)} \\ \frac{\alpha^{(4)}-\mathbf{r}^{(4)}}{\beta^{(4)}-\alpha^{(4)}} i, & \mathbf{r}^{(4)}<\alpha^{(4)}\end{cases}
$$

$\mathbf{G}_{5}$ Tests with one finite reference range:

Medical tests, which are classified in this group, are not well known to wide population. A size of cancer and a $T$-score used for rating of Bone-Mass-Measurement are the best known tests from this group.

Let $\mathbf{r}^{(\mathbf{5})}$ be a result of test from this group with a reference range $\gamma^{(5)}$ (both the result and the reference range are measured in measure unit $\mathcal{U}$ ). An estimate of the result $\mathbf{r}^{(\mathbf{5})}$ is

$$
e^{5}\left(\mathbf{r}^{(\mathbf{5})}\right)= \begin{cases}0, & \text { negative result, } \\ \left(\mathbf{r}^{(\mathbf{5})}-\gamma^{(5)}\right) / 1 \mathcal{U}, & \text { positive result } \mathbf{r}^{(\mathbf{5})}>\gamma^{(5)}, \\ i\left(\gamma^{(5)}-\mathbf{r}^{(\mathbf{5})}\right) / 1 \mathcal{U}, & \text { positive result } \mathbf{r}^{(\mathbf{5})}<\gamma^{(5)}\end{cases}
$$

It is evident that modules of estimates $(2.1,2.3,2.4,2.5,2.6)$ of least intensive disorders in tests from the groups $G_{1}-G_{5}$ are the least of modules of all 
possible estimates of positive results of the corresponding test. Moreover, a negative result in any of tests from the groups $G_{1}, G_{2}, G_{4}, G_{5}$ is estimated with 0 . Specially, the most intensive disorders in tests from the groups $G_{1}$ and $G_{2}$ are estimated with 1 . The least intensive disorders in the tests from the group $G_{2}$ are estimated with 0.5. Estimates of a result $r_{0}^{(4)}$ of a tests from the group $G_{4}$ with reference ranges $\alpha_{0}^{(4)}$ and $\beta_{0}^{(4)}$ are equal to the corresponding estimates of the result $\mathbf{r}_{0}^{*}=\left(\mathbf{r}_{0}^{(4)}-\alpha_{0}^{(4)}\right) /\left(\beta_{0}^{(4)}-\alpha_{0}^{(4)}\right)$ of the test with reference ranges 0 and 1 . From the same reason, the estimate of result $\mathbf{r}^{(\mathbf{5})}{ }_{0}$ of test with a reference range $\gamma_{0}^{(5)}$ from the group $G_{5}$ is equal to the estimate of result $\mathbf{r}_{0}^{* *}=\left(\mathbf{r}_{0}^{(5)}-\gamma_{0}^{(5)}\right) / 1 \mathcal{U}$ of test with reference range 0 . In this way, we proved that medical tests from the same group are coherent. For this reason, the corresponding estimates of results of these tests can be linearly combined.

It is not suitable to compare disorder-intensities of results of tests from different groups because different groups of tests correspond to different dimensions of the patient's status.

Proposition 2.1: Results of tests from any of previous defined five groups are coherent. The estimates $(2.1,2.3,2.4,2.5,2.6)$ of results of different tests from the same group may be linearly combined.

Definition 2.1: Let $e_{1}^{k}, \ldots, e_{n_{k}}^{k}$ be estimates of all results in tests from the group $G_{k}, k=1, \ldots, 5$ and let be $I_{0}^{k}=\left\{i_{1}^{k}, \ldots, i_{r}^{k}\right\} \subseteq\left\{1, \ldots, n_{k}\right\}=I^{k}$.

- The sum $\mathcal{E}_{I_{0}^{k}}=\sum_{u=1}^{r} e_{i_{u}^{k}}^{k}$ is the estimate of the results from the group $I_{0}^{k}$,

- The sum $\mathcal{E}_{I^{k}}=\sum_{u=1}^{n_{k}} e_{u}^{k}$ is the estimate of all results from the group $G_{k}$,

- The modulo $\mathcal{E}_{I_{0}^{k}}^{A b s}=\left|\mathcal{E}_{I_{0}^{k}}\right|$ is the absolute estimate of the estimate $\mathcal{E}_{I_{0}^{k}}$,

- The square root $\mathcal{E}_{I_{0}^{k_{1}, \ldots, I_{0}}}^{A b s}=\sqrt{\sum_{u=1}^{r}\left(\mathcal{E}_{I_{0}^{k_{u}}}^{A b s}\right)^{2}}$ is the estimate of tests from the (sub)groups $I_{0}^{k_{1}}, \ldots, I_{0}^{k_{r}}$,

- The square root $\mathcal{E}=\sqrt{\sum_{u=1}^{5} \mathcal{E}_{I^{u}}^{A b s}}$ is the estimate of the complete status of patient.

2.2. Graphical analysis of previously estimated results. Human organisms are stable biochemical mixtures. Organisms aim to preserve their biochemical stabilities. Any aberration from the stability, doctors recognize as a disease.

Because our organisms protect us of diseases, any disorder in the biochemical stability appears insensibly. Trough the time, the state gets worse rapidly. At some moment, the increasing of an intensity of disorder becomes to slow down. At some moment, there are not changes of status. After that, status may start to improve or to exacerbate. A preferment of a status, as an exacerbation, starts slowly. The preferment becomes faster and faster. After some period, it slows down to the stability. Graphically, the changes of result-estimates from the day 1 to day 7 (the complete previously explained periods) have the form as in Figure 1 .

Functions which connect results on the previous figure are polynomials. Results $\mathbf{r}_{1}$ and $\mathbf{r}_{2}$ in a test realized at the times $t_{1}$ and $t_{2}$ are connected with the function 


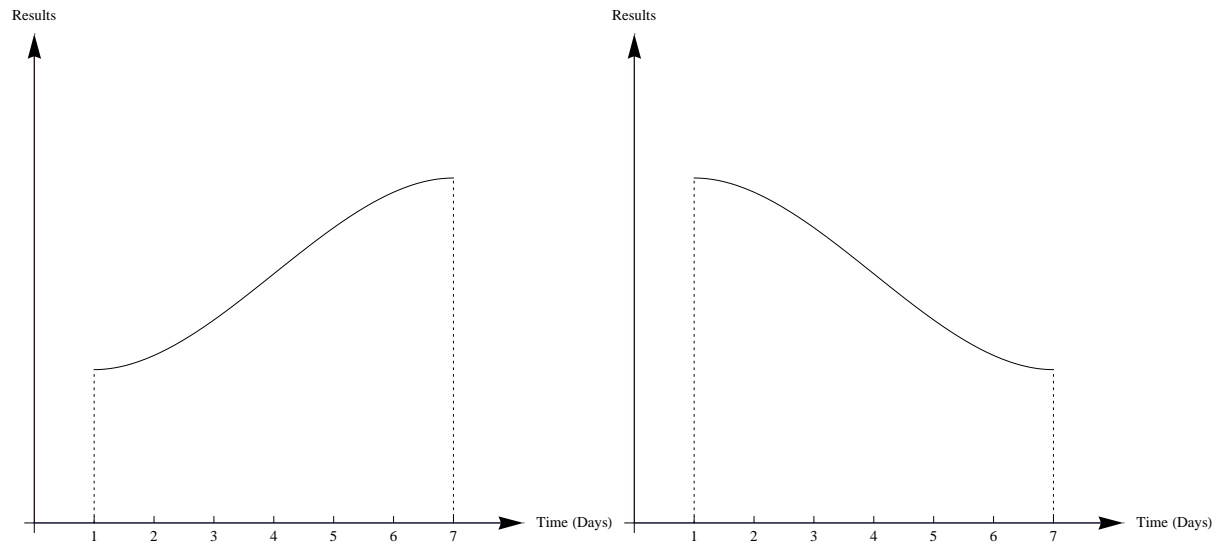

FiguRE 1. Exacerbation and preferment of a state in a test

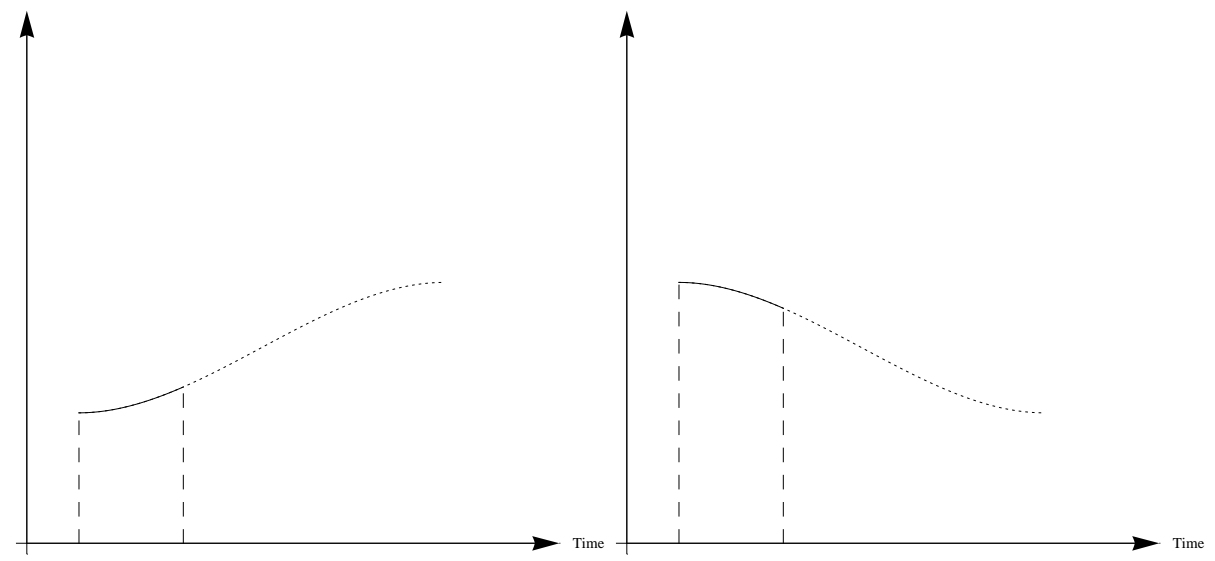

Figure 2. Changes in chronic diseases

$$
A(t)=-2 \frac{\mathbf{r}_{2}-\mathbf{r}_{1}}{\left(t_{2}-t_{1}\right)^{3}}\left(t-t_{1}\right)^{2}\left(t-\frac{3 t_{2}-t_{1}}{2}\right)+\mathbf{r}_{1} .
$$

Two types of diseases exist (chronic and acute diseases). The function $A(t)$ approximately connects results $\mathbf{r}_{1}$ and $\mathbf{r}_{2}$ which changes are caused by an acute disease.

Changes of results caused by a chronic disease are slow and they are not very intensive at the start. When changes become intensive, the situation is very dangerous. For this reason, doctors have scheduled relatively frequent check inspections for the reason of quick and adequate reaction. Graphically, changes of these results are presented above (see Figure 2). 
The black lines, between dashed verticals, are like graphs of quadratic functions. So, these results are connected with the function [17]

$$
C(t)=\frac{\mathbf{r}_{2}-\mathbf{r}_{1}}{\left(t_{2}-t_{1}\right)^{2}}\left(t-t_{1}\right)^{2}+\mathbf{r}_{1} .
$$

The third case, when it is not known which type of a disease caused changes of results $\mathbf{r}_{1}$ and $\mathbf{r}_{2}$, is also important. In that case, the results are connected linearly with the function

$$
L(t)=\frac{\mathbf{r}_{2}-\mathbf{r}_{1}}{t_{2}-t_{1}}\left(t-t_{1}\right)+\mathbf{r}_{1}
$$

For the functions $L(t), C(t), A(t)$, we obtain

$$
L\left(t_{1}\right)=C\left(t_{1}\right)=A\left(t_{1}\right)=\mathbf{r}_{1} \quad \text { and } \quad L\left(t_{2}\right)=C\left(t_{2}\right)=A\left(t_{2}\right)=\mathbf{r}_{2} .
$$

Generally, results $\mathbf{r}_{1}$ and $\mathbf{r}_{2}$ of a test realized at the times $t_{1}$ and $t_{2}$ are connected with the function

$$
F(t)=(-2)^{\beta} \frac{\mathbf{r}_{2}-\mathbf{r}_{1}}{\left(t_{2}-t_{1}\right)^{\alpha+\beta}}\left(t-t_{1}\right)^{\alpha}\left(t-\frac{3 t_{2}-t_{1}}{2}\right)^{\beta}+\mathbf{r}_{1}
$$

for $(\alpha, \beta) \in\{(1,0),(2,0),(2,1)\}$. In the case of $(\alpha, \beta)=(1,0)$ the function $F$ is linear (2.9). In the case of $(\alpha, \beta)=(2,0)$ the function $F$ is quadratic (2.8). In the case of $(\alpha, \beta)=(2,1)$ the function $F$ is the function $(2.7)$.

Let $t_{0} \in\left[t_{1}, t_{2}\right]$ be some moment. Values $L\left(t_{0}\right), C\left(t_{0}\right), A\left(t_{0}\right)$ are the approximated results of the corresponding test. The corresponding estimates $(2.1,2.3,2.4,2.5,2.6)$ of the results $L\left(t_{0}\right), C\left(t_{0}\right), A\left(t_{0}\right)$ are the approximated estimates of the approximated results.

Remark 2.1: Estimates of the results of tests from the group $G_{1}$ are numerically expressed results of these tests.

Remark 2.2: Let a patient walks $d_{1}$ kilometers at the time $t_{1}$ and let he walks $d_{2}$ kilometers with a help at the time $t_{2}$. In the case of the patient walks alone to a time $t_{0}^{(3)} \in\left(t_{1}, t_{2}\right)$ than the corresponding function $(2.7,2.8,2.9)$, which connects the results, should be restricted on the segments $\left[t_{1}, t_{0}^{(3)}\right]$ and $\left[t_{0}^{(3)}, t_{2}\right]$. If it is known a distance $d_{0}^{3}$ which patient walked alone at the time $t_{0}^{(3)}$, the restrictions should have the corresponding forms (2.4) for the corresponding results $d_{1}, d_{0}, d_{2}$.

Remark 2.3: Let $\mathbf{r}_{1}^{*}$ and $\mathbf{r}_{2}^{*}$ be transformed results as above of a test from the group $G_{4}$ realized at times $t_{1}$ and $t_{2}$, respectively and let these results be connected with the corresponding function $f(t)$. The corresponding function $\widetilde{F}(t)$ which connects estimates of the results in this test is

$$
\widetilde{F}(t)= \begin{cases}0, & 0 \leq f(t) \leq 1 \\ f(t)-1, & f(t)>1 \\ -f(t) i, & f(t)<0\end{cases}
$$


Moments when the function $\widetilde{F}(t)$ changes the form $(0 \longleftrightarrow f(t), 0 \longleftrightarrow f(t) i)$ are solutions of the equations

$$
f(t)=1 \quad \text { and } \quad f(t)=0
$$

on the set $\left[t_{1}, t_{2}\right]$, respectively.

Analogous process should be realized for results in tests from the group $G_{5}$. Namely, in the case of the results $\mathbf{r}_{1}^{* *}$ and $\mathbf{r}_{2}^{* *}$ are connected with the corresponding function $g(t)$, the estimates of these results are connected with the function

$$
\widetilde{G}(t)= \begin{cases}0, & \text { negative result }, \\ g(t), & g(t)>0 \\ g(t) i, & g(t)<0\end{cases}
$$

A time $t_{0}^{(5)}$, when the function $\widetilde{G}(t)$ changes the form, is solution of the equation

$$
g(t)=0 .
$$

Times $t_{0}^{(3)}, t_{0_{1}}^{(4)}, t_{0_{2}}^{(4)}, t_{0}^{(5)}$ from the Remarks 2.2 and 2.3are the critical times (of medical tests).

Let times

$$
t_{1}<\ldots<t_{q+1}
$$

be times when all control exams are realized together with the corresponding critical times. Let also

$$
\mathbf{r}_{1}^{(u)(k)}, \ldots, \mathbf{r}_{q+1}^{(u)(k)}, k=1, \ldots, 5, u=1, \ldots, n_{k},
$$

be approximate results of the $u$-th test from the $k$-th group and let the function $f_{r}^{(u)(k)}(t), r=1, \ldots, q$, functionally connects the estimates of the results $\mathbf{r}_{r}^{(u)(k)}$ and $\mathbf{r}_{r+1}^{(u)(k)}$. The coordinate matrix curve

$$
\mathcal{T}^{(u)(k)}(t)=\left[\begin{array}{lll}
f_{1}^{(u)(k)}(t) & \ldots & f_{q}^{(u)(k)}(t)
\end{array}\right]
$$

of the type $1 \times q$ defined on the set $\left[t_{1}, t_{2}\right] \times\left[t_{2}, t_{3}\right] \times \ldots \times\left[t_{q}, t_{q+1}\right]$ functionally connects the estimates of the approximated results of the $u$-th tests from the $k$-th group.

To estimate stabilities of the results of different medical tests and of special groups of tests at a time $t_{0}$, we will use the corresponding areas generated with the graph of the corresponding function, the $x$-axis and the vertical lines $x=t_{1}$ and $x=t_{0}$. Let $\mathcal{T}^{(u)(k)}$ be a coordinate matrix curve of the type $1 \times q$. The coordinate matrix curve

$$
\mathcal{C}_{\mathcal{T}^{(u)(k)}}^{(u)(k)}(t)=\lim _{u \rightarrow t} \frac{1}{u-t_{1}}\left[\begin{array}{lll}
\mathcal{A}_{\mathcal{T}^{(u)(k)}}^{t_{1}, \ldots, t_{q+1} ; 1}(u) & \ldots & \mathcal{A}_{\mathcal{T}^{(u)(k)}}^{t_{1}, \ldots, t_{q+1} ; q}(u)
\end{array}\right]
$$

of the type $1 \times q$ defined on the set $\left[t_{1}, t_{2}\right] \times\left[t_{2}, t_{3}\right] \times \ldots \times\left[t_{q}, t_{q+1}\right]$ is the estimate of the history of these results at the time $t$.

The coordinate matrix curves: 


$$
\begin{aligned}
& \mathcal{T}_{I_{0}^{k}}(t)=\left[\begin{array}{lll}
\sum_{u=1}^{r} f_{1}^{\left(i_{u}^{k}\right)(k)}(t) & \ldots & \sum_{u=1}^{r} f_{q}^{\left(i_{u}^{k}\right)(k)}(t)
\end{array}\right], \\
& \mathcal{T}_{I^{k}}(t)=\left[\begin{array}{lll}
\sum_{u=1}^{n_{k}} f_{1}^{(u)(k)}(t) & \ldots & \sum_{u=1}^{n_{k}} f_{q}^{(u)(k)}(t)
\end{array}\right], \\
& \mathcal{T}_{I_{0}^{k}}^{A b s}(t)=\left[\left|\sum_{u=1}^{r} f_{1}^{\left(i_{u}^{k}\right)(k)}(t)\right| \quad \ldots|| \sum_{u=1}^{r} f_{q}^{\left(i_{u}^{k}\right)(k)}(t) \mid\right], \\
& \mathcal{T}_{I_{0}^{k_{1}}, \ldots, I_{0}^{k_{s}}}^{A b s}(t)=\left[\begin{array}{lll}
\sqrt{\sum_{v=1}^{s}\left|\sum_{u=1}^{r} f_{1}^{(i v)(v)}(t)\right|^{2}} & \cdots \sqrt{\sum_{v=1}^{s}\left|\sum_{u=1}^{r} f_{q}^{\left(i_{u}^{v}\right)(v)}(t)\right|^{2}}
\end{array},\right. \\
& \mathcal{T}(t)=\left[\begin{array}{lll}
\sqrt{\sum_{v=1}^{5}\left|\sum_{u=1}^{n_{v}} f_{1}^{(u)(v)}(t)\right|^{2}} & \cdots & \sqrt{\sum_{v=1}^{s}\left|\sum_{u=1}^{n_{v}} f_{q}^{(u)(v)}(t)\right|^{2}}
\end{array}\right]
\end{aligned}
$$

functionally connect the above defined (approximated) estimates $E_{I_{0}}^{k}, E_{I^{k}}, E_{I_{0}^{k}}^{A b s}$, $E_{I_{0}^{k_{1}}, \ldots, I_{0}^{k_{r}}}^{A b s}, E$ of results at the times $t_{1}, \ldots, t_{q+1}$ with regard to the corresponding functions $A(t), C(t), L(t)$ from the equations $(2.7,2.8,2.9)$. The coordinate matrix curves

$$
\begin{aligned}
& \mathcal{C}_{I_{0}^{k}}^{t_{1}, \ldots, t_{q+1}}(t)=\lim _{u \rightarrow t} \frac{1}{u-t_{1}}\left[\begin{array}{ccc}
\mathcal{A}_{\mathcal{T}_{I_{0}^{k}}}^{t_{1}, \ldots, t_{q+1} ; 1}(u) & \ldots & \mathcal{A}_{\mathcal{T}_{I_{0}^{k}}}^{t_{1}, \ldots, t_{q+1} ; q}(u)
\end{array}\right], \\
& \mathcal{C}_{I^{k}}^{t_{1}, \ldots, t_{q+1}}(t)=\lim _{u \rightarrow t} \frac{1}{u-t_{1}}\left[\begin{array}{lll}
\mathcal{A}_{\mathcal{T}^{k}}^{t_{1}, \ldots, t_{q+1} ; 1}(u) & \ldots & \mathcal{A}_{\mathcal{T}_{I^{k}}^{t_{1}, \ldots, t_{q+1} ; q}(u)}
\end{array}\right], \\
& \mathcal{C}_{I^{k}}^{A b s ; t_{1}, \ldots, t_{q+1}}(t)=\lim _{u \rightarrow t} \frac{1}{\left|u-t_{1}\right|}\left[\begin{array}{lll}
\left|\mathcal{A}_{\mathcal{T}^{k}}^{t_{1}, \ldots, t_{q+1} ; 1}(u)\right| & \cdots & \left|\mathcal{A}_{\mathcal{T}^{I^{k}}}^{t_{1}, \ldots, t_{q+1} ; q}(u)\right|
\end{array}\right],
\end{aligned}
$$

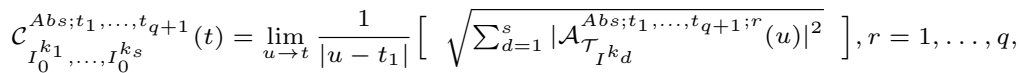

$$
\begin{aligned}
& \mathcal{C}^{t_{1}, \ldots, t_{q+1}}(t)=\lim _{u \rightarrow t} \frac{1}{\left|u-t_{1}\right|}\left[\sqrt{\sum_{d=1}^{5} \mid \mathcal{A}_{\left.\mathcal{T}_{I}{ }^{d}{ }^{A s ; t_{1}, \ldots, t_{q+1} ; r}(u)\right|^{2}}^{2}}\right], r=1, \ldots, q,
\end{aligned}
$$

defined on the set $\left[t_{1}, t_{2}\right] \times\left[t_{2}, t_{3}\right] \times \ldots \times\left[t_{q}, t_{q+1}\right]$ functionally connect the history of approximated estimates of the results.

Let a coordinate matrix curve $\mathcal{T}(t)=\left[\tau_{1}(t) \ldots \tau_{q}(t)\right]$, defined on the set $\left[t_{1}, t_{2}\right] \times \ldots \times\left[t_{q}, t_{q+1}\right]$, connects estimates of a group of medical results and let $\mathcal{C}(t)=\left[c_{1}(t) \ldots c_{q}(t)\right]$ be the corresponding coordinate matrix curve obtained with regard to the curve $\mathcal{T}(t)$. Denote by

$$
c= \begin{cases}\left|\tau_{1}\left(t_{1}\right)\right|, & \tau_{1}\left(t_{1}\right) \neq 0, \\ 1, & \tau_{1}\left(t_{1}\right)=0, \\ \rho>0, & \text { in a special case }\end{cases}
$$

a comparison value (comparator) of these results. The coordinate matrix curves

$$
\widetilde{\mathcal{T}}(t)=\frac{1}{c} \mathcal{T}(t) \quad \text { and } \quad \widetilde{\mathcal{C}}(t)=\frac{1}{c} \mathcal{C}(t)
$$

are the time-analysis-curve (TAC) and the complete-analysis-curve (CAC).

Remark 2.4: If a function $\left|\tau_{k}(t)\right|, k=1, \ldots, q$, obtained from the curve $\mathcal{T}(t)$ is a decreasing/increasing function then the corresponding state improves/worsens in the period $\left[t_{k}, t_{k+1}\right]$. 
If a function $\left|c_{k}(t)\right|, k=1, \ldots, q$, obtained from the curve $\mathcal{C}(t)$ is a decreasing/increasing one then the corresponding stability of the state improves/worsens in the period $\left[t_{k}, t_{k+1}\right]$.

2.3. Properties of the method. The following Lemma holds.

Lemma 1: The $k$-th element of a CAC which connects non-absolute estimates

$$
E=\left(E_{1}, \ldots, E_{q+1}\right)
$$

of test results realized at the times $T=\left(t_{1}, \ldots, t_{q+1}\right)$ is

$$
c_{k}(t)=\frac{1}{c} \lim _{u \rightarrow t} \frac{b_{k}(u)+\sum_{r=1}^{k-1} \gamma_{r}\left(\delta_{r} E_{r}+E_{r+1}\right)\left(t_{r+1}-t_{r}\right)}{u-t_{1}},
$$

for

$$
b_{k}(t)=\zeta_{k} \frac{E_{k+1}-E_{k}}{\left(t_{k+1}-t_{k}\right)^{\alpha_{k}+\beta_{k}}}\left(t-t_{k}\right)^{\alpha_{k}+1}\left(t+t_{k}-2 t_{k+1}\right)^{\beta_{k}}+E_{k}\left(t-t_{k}\right)
$$

with $\left(\alpha_{k}, \beta_{k}\right) \in\{(1,0),(2,0),(2,1)\}$ and

$$
\gamma_{r}=\frac{1}{\alpha_{r}-\beta_{r}+1}, \quad \delta_{r}=\alpha_{r}-\beta_{r}, \quad \zeta_{r}=\frac{1}{\alpha_{r}-5 \beta_{r}+1} .
$$

Proof. Let us prove this lemma for linearly connected results. Proofs of all other cases are analogical.

Let $\tau(t)=\left[\tau_{k}(t)\right]$ be the TAC which connects the estimates of results at the times $t_{1}, \ldots, t_{q+1}$. Functions $\tau_{k}(t), k=1, \ldots, q$, are

$$
\tau_{k}(t)=\frac{E_{k+1}-E_{k}}{t_{k+1}-t_{k}}\left(t-t_{k}\right)+E_{k} .
$$

The function $c_{k}(t)$ in the corresponding $\mathrm{CAC}$ is

$$
c_{k}(t)=\lim _{u \rightarrow t} \frac{\sum_{p=1}^{k-1} \int_{t_{p}}^{t_{p+1}} \tau_{p}(\rho) d \rho}{u-t_{1}}+\lim _{u \rightarrow t} \frac{\int_{t_{k}}^{u} \tau_{k}(\rho) d \rho}{u-t_{1}} .
$$

It holds the equality

$$
\begin{aligned}
& \int_{t_{p}}^{t_{p+1}} \tau_{p}(\rho) d \rho=\int_{t_{p}}^{t_{p+1}}\left(\frac{E_{p+1}-E_{p}}{t_{p+1}-t_{p}}\left(u-t_{p}\right)+E_{p}\right) d u=\frac{1}{2}\left(E_{p+1}+E_{p}\right)\left(t_{p+1}-t_{p}\right) . \\
& \int_{t_{k}}^{t} \tau_{k}(\rho) d \rho=\frac{1}{2} \frac{E_{k+1}-E_{k}}{t_{k+1}-t_{k}}\left(t-t_{k}\right)^{2}+E_{k}\left(t-t_{k}\right) .
\end{aligned}
$$

These equalities, involved in the equation (2.34), prove this lemma.

Organisms are less or more resistant on any therapy. For this reason, any cure should be applied through as short as possible period. In the cases of acute diseases, therapies have been applied at most few months. Otherwise, therapies for chronic diseases are the therapies which have been applied in whole lives of patients. 
Doctors need to know what happens with statuses of different patients who are cured with different therapies. Different periods are necessary for cures with different therapies. The following statements will equalize different of these periods.

Proposition 2.2: Let $\tau(t)=\left[\begin{array}{llll}\tau_{1}(t) & \ldots & \tau_{q}(t)\end{array}\right]$ and $\mathcal{C}(t)=\left[\begin{array}{llll}c_{1}(t) & \ldots & c_{q}(t)\end{array}\right]$ be $\mathrm{TAC}$ and $\mathrm{CAC}$ which functionally connect the estimates $E_{1}, \ldots, E_{q+1}$ of the approximate results $\mathbf{r}=\left(\mathbf{r}_{1}, \ldots, \mathbf{r}_{q+1}\right)$ at the times $t_{1}, \ldots, t_{q+1}$ and let $\alpha \in \mathbb{R} \backslash\{0\}, \beta \in \mathbb{R}$ be random constants. Let also the coordinate matrix curves $\tau^{*}(t)=\left[\begin{array}{llll}\tau_{1}^{*}(t) & \ldots & \tau_{q}^{*}(t)\end{array}\right]$ and $\mathcal{C}^{*}=\left[\begin{array}{llll}c_{1}^{*}(t) & \ldots & c_{q}^{*}\end{array}\right]$ connect the estimates $E_{1}, \ldots, E_{q+1}$ of the results of the corresponding tests realized at the times $\widetilde{t}_{k}=\alpha t_{k}+\beta, k=1, \ldots, q+1$. It holds

$$
\tau_{k}^{*}\left(t^{*}\right)=\tau_{k}(t) \quad \text { and } \quad c_{k}^{*}\left(t^{*}\right)=c_{k}(t)
$$

for any $t$.

Proof. We will prove this proposition just for the case of linear connections. The other cases can be proved analogically.

Let be $t_{0} \in\left[t_{k}, t_{k+1}\right]$, for the moments $t_{k}, t_{k+1}$ when the test was realized. Let $\mathbf{r}_{k}$ and $\mathbf{r}_{k+1}$ be the results from these control exams. For any of the types $(2.7,2.8,2.9)$ of the function $f_{k}(x)$ which functionally connect $\mathbf{r}_{k}$ and $\mathbf{r}_{k+1}$, we have

$$
f_{k}^{*}(\alpha x+\beta)=f_{k}(x) .
$$

Really, if the functions $f_{k}$ and $f_{k}^{*}$ have the form (2.9) it holds

$$
f_{k}^{*}\left(t^{*}\right)=\frac{\mathbf{r}_{k+1}-\mathbf{r}_{k}}{\left(a t_{k+1}+b\right)-\left(a t_{k}+b\right)}\left((a t+b)-\left(a t_{k}+b\right)\right)+\mathbf{r}_{1}=f_{k}(t) .
$$

From this equality, it directly holds $\tau_{k}^{*}\left(t^{*}\right)=\tau_{k}(t)$.

Moreover, it holds

$$
\int_{t_{k}^{*}}^{t_{0}^{*}} f_{k}^{*}\left(t^{*}\right) d t^{*}=\left|\begin{array}{ll}
\text { Change: } & u=\frac{t^{*}-b}{a}=t \in\left[t_{k}, t_{k+1}\right] \\
& d u=\frac{1}{a} d t^{*} \\
& u_{k}=\frac{t_{k}^{*}-b}{t_{0}}=t_{k} \\
& u_{0}=\frac{t_{0}^{*}-b}{a}=t_{0}
\end{array}\right|=\int_{t_{k}}^{t_{0}} f_{k}(t) d t .
$$

This equality proves the validity of $c_{k}^{*}\left(t^{*}\right)=c_{k}(t)$. The other two cases may be proved analogically.

Corollary 2.1: If control exams are realized at the times $\mathcal{T}=\left(t_{1}, \ldots, t_{q+1}\right)$, the results may be equivalently analyzed as results from the control exams at the times $\widetilde{\mathcal{T}}=\left(\tilde{t}_{1}, \ldots, \tilde{t}_{q+1}\right)$ such that

$$
\left(t_{2}-t_{1}\right): \ldots:\left(t_{q+1}-t_{q}\right)=\left(\tilde{t}_{2}-\tilde{t}_{1}\right): \ldots:\left(\tilde{t}_{q+1}-\tilde{t}_{q}\right) .
$$


We want to examine what are the measure characteristics of the estimates $(2.1,2.3,2.4,2.5,2.6)$. The following theorem holds.

Theorem 2.1: The estimates $\mathcal{E}_{I_{0}^{k}}$ and $\mathcal{E}_{I^{k}}$ from Definition 2.1 are $\sigma$-additive complex measures. The estimates $\mathcal{E}_{I_{0}^{k}}^{A b s}, \mathcal{E}_{I_{0}^{k_{1}}, \ldots, I_{0}^{k_{r}}}^{A b s}, \mathcal{E}$ from Definition 2.1 are pseudoadditive measures.

Proof. The first part of this theorem evidently holds (because of the caused linearity from the definitions of $\mathcal{E}_{I_{0}^{k}}$ and $\mathcal{E}_{I^{k}}$ ).

To prove the second part of this theorem, we need to notice that $\mathcal{E}_{\emptyset}^{A b s}=0$. For this reason, any $\mathcal{E}_{I_{0}^{k_{1}}, \ldots, I_{0}^{k_{r}}}^{A b s}$ has the form

$$
\mathcal{E}_{I_{0}^{k_{1}, \ldots, I_{0} k_{r}}}^{A b s} \equiv \mathcal{E}_{I_{0}^{1}, \ldots, I_{0}^{5}}^{A b s},
$$

for some $I_{0}^{p}=\emptyset$. It also holds

$$
\mathcal{E}_{I_{0}^{k_{1}}, \ldots, I_{0}^{k_{r}}}^{A b s}=\sqrt{\left|\mathcal{E}_{I_{0}^{k_{1}}}\right|^{2}+\ldots+\left|\mathcal{E}_{I_{0}^{k_{r}}}\right|^{2}} \equiv \sqrt{\left|\mathcal{E}_{I_{0}^{1}}\right|^{2}+\ldots+\left|\mathcal{E}_{I_{0}}^{5}\right|^{2}} .
$$

For the estimates $\mathcal{E}_{I_{0}^{1}, \ldots I_{0}^{5}}^{A b s}$ and $\mathcal{E}_{J_{0}^{1}, \ldots J_{0}^{5}}^{A b s}$, the binary operation $*$ is defined as:

$$
\mathcal{E}_{I_{0}^{1}, \ldots, I_{0}^{5}}^{A b s} * \mathcal{E}_{J_{0}^{1}, \ldots, J_{0}^{5}}^{A b s}:=\mathcal{E}_{I_{0}^{1} \cup J_{0}^{1}, \ldots, I_{0}^{5} \cup J_{0}^{5}}^{A b} .
$$

The estimate of the union of disjoint sets of medical tests is evidently additive by the operation $*$. Furthermore, because $f(x)=\sqrt{x}$ is the increase and continuous function we conclude that if $I_{1}^{k} \subset I_{2}^{k} \subset \ldots$ and $I_{0}^{k} \underset{n \rightarrow \infty}{\longrightarrow} I_{0}^{k}, k=1, \ldots, 5$, then the

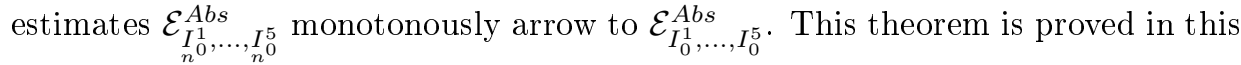
way.

Corollary 2.2: The coordinate matrix curves which functionally connect estimates have the same measure properties as the estimates which they connect.

2.4. Algorithms. Now the algorithms can be presented.

\section{Algorithm 1: OPTCAC}

This algorithm numerically and graphically estimates patient's state of health. States trough time will be compared with the special state.

Inputs: Results $\mathbf{r}_{u}^{k}, u=1, \ldots, n_{k}, k=1, \ldots, 5$, the corresponding reference ranges $\alpha_{u}^{4}, \beta_{u}^{4}, \gamma_{v}^{5}$, the times $T=\left\{t_{1}, \ldots, t_{q+1}\right\}$ with involved the corresponding critical ones, the subgroups of tests $I_{0}^{k}$ and special times $\widetilde{T}=\left(t_{1}^{0}, \ldots, t_{s}^{0}\right)$;

Step 1: Apply the equations $(2.1,2.3,2.4,2.5,2.6)$ to estimate the results $\mathbf{r}_{k}^{g}$;

Step 2: From the corresponding functions (2.7), find the critical times $t_{0}^{(3)}, t_{0_{1}}^{(4)}, t_{0_{2}}^{(4)}, t_{0}^{(5)}$ if they exist and functionally connect the results with coordinate matrix curves $\left(M_{\left(k_{g}\right)}^{g}(t)\right)_{g=1}^{5}$;

Step 3: From the curves $M_{\left(k_{q}\right)}^{g}(t)$, find the corresponding TACs and CACs;

Step 4: Find the corresponding TACs and CACs of combinations of tests;

Step 5: Polynomials $p(t)$ with complex coefficients in the TACs and CACs from the Step 4 , transform to $(\Re(p)(t),-\Im(p)(t))$;

Step 7: Plot graphs $\Gamma_{u}$ of transformed TACs and CACs from the Step 5;

Output: Estimates at special times $\widetilde{T}$ and graphs $\Gamma_{u}$. 
By using the following algorithm, it may be estimated a status of a group of patients.

Algorithm 2: GPTCAC

This algorithm estimates a status of a group consisted of $n$ patients. The aim of this algorithm is to find expectations (meanings) of compared and non-compared estimates such as to analyze these expectations.

Inputs: Corresponding results of $n$ patients, times of control exams together with the critical times $T=\left(t_{1}, \ldots, t_{q+1}\right)$, the conditions $c_{1}, \ldots, c_{m}$ in the forms "preferments/exacerbations are registered at at least/at most $c_{u} \cdot n$ patients" and the times $\mathcal{T}=\left(t^{1}, \ldots, t^{s}\right)$ when results are important;

Step 1: Apply algorithm OPTCAC and obtain the corresponding coordinate matrix curves $M^{k}(t), \tau^{k}(t), \mathcal{C}(t), k=1, \ldots, n$ defined on the set $\mathbb{D}=\left[t_{1}, t_{2}\right] \times \ldots \times\left[t_{q}, t_{q+1}\right] ;$

Step 2: Form the coordinate matrix curves

$$
M(t)=\left[\begin{array}{c}
M^{1}(t) \\
\vdots \\
M^{n}(t)
\end{array}\right], \quad \tau(t)=\left[\begin{array}{c}
\tau^{1}(t) \\
\vdots \\
\tau^{n}(t)
\end{array}\right], \quad \mathcal{C}(t)=\left[\begin{array}{c}
\mathcal{C}^{1}(t) \\
\vdots \\
\mathcal{C}^{n}(t)
\end{array}\right]
$$

Step 3: Find the mid-para-curves

$$
\mathcal{M}_{M}(t), \quad \mathcal{M}_{\tau}(t), \quad \mathcal{M}_{\mathcal{C}}(t)
$$

Step 4: Form TAC $\widetilde{\tau}(t)$ and $\mathrm{CAC} \widetilde{\mathcal{C}}(t)$ of the coordinate matrix curve $\mathcal{M}_{M}(t)$ obtained into the equation $(2.40)$

Step 5: Find the approximated estimates $\left(\mathcal{Z}\left(t^{k}\right)\right)_{k=1}^{s}$, for

$$
\mathcal{Z} \in\left\{\mathcal{M}_{M}, \mathcal{M}_{\tau}, \mathcal{M}_{\mathcal{C}}, \widetilde{\tau}, \widetilde{\mathcal{C}}\right\}
$$

Step 6: Find the frequencies $f_{k}=n_{k} / n$, for the conditions $c_{k}, k=1, \ldots, m$ where $n_{k}$ is the number of patients which satisfy this condition;

Step 7: Form the graphs $\Gamma_{u}$ of the coordinate matrix curves obtained in the Step 3 and Step 4;

Step 8: Recursively apply this algorithm on special subpopulations of the population whose results are tested in the previous steps if it is necessary;

Outputs: All approximated estimates at the times $\mathcal{T}$, all frequencies $f_{k}$ and all formed graphs $\Gamma_{k}$.

Remark 2: Coordinate matrix curve $\mathcal{M}_{M}(t)$ from the Step 3 in GPTCAC analyzes expected effects of a therapy on a whole population.

Remark 3: Algorithm GPTCAC is a finite one because there is only finite number of sub-populations of any $n$-patients size population.

Remark 4: Parts of graphs into GPTCAC where conditions $c_{1}, \ldots, c_{k}$ are satisfied should be formed with different styles of the other parts of these graphics where it is not the case.

Remark 5: Algorithm GPTCAC should be applied on different disjoint subgroups formed of patients whose status-estimates are closely same numbers (it is a principle in statistical analysis of medical data).

Algorithm 3: CTCAC

This algorithm compares effects of different therapies such the effects of different dozes of the same therapy. Using the Proposition 2.2 and Corollary 2.1, this algorithm may compare the results of different experiments. 
Inputs: Coordinate matrix curves

$$
\mu^{1}(t) \quad \ldots \quad \mu^{k}(t)
$$

which functionally connect the estimates of corresponding results in therapies with the times of control tests $\left(t_{v}^{u}\right), u=1, \ldots, k, v=1, \ldots, q_{u}+1$ and special times $\mathcal{T}=\left(t_{0}^{1}, \ldots, t_{0}^{k}\right)$. The curve $\mu^{p}(t)$ is defined on the set $\mathbb{D}^{p}=\left[t_{1}^{p}, t_{2}^{p}\right] \times \ldots \times\left[t_{q_{p}}^{p}, t_{q_{p}+1}^{p}\right] ;$

Step 1: Any of the times $t_{0}^{p}$ transform to $\tilde{t}_{0}^{p}=\left(t_{0}^{p}-t_{1}^{p}\right) /\left(t_{q_{p}+1}^{p}-t_{1}^{p}\right)$;

Step 2: The coordinate matrix curves $\mu^{p}(t)$ transform to the corresponding coordinate matrix curves $\widetilde{\mu}^{p}(t)$ defined on the sets $\mathbb{D}^{p}=\left[\tilde{t}_{1}^{p}, \tilde{t}_{2}^{p}\right] \times \ldots \times\left[\tilde{t}_{q_{p}}^{p}, 1\right]$;

Step 3: Compare the approximated estimates

$$
\tilde{\mu}^{1}\left(\tilde{t}_{0}^{1}\right) \quad \ldots \widetilde{\mu}^{k}\left(\tilde{t}_{0}^{k}\right) ;
$$

Outputs: The corresponding conclusions derived from the comparisons realized at the Step 3 .

\section{Conclusion}

A novel approach to medical data analysis was presented in this paper. This way of analysis makes possible for anybody to see numerical estimates of results and to understand what really happened. Computer graphics is useful here because numerical estimates of results are connected graphically based on the passed time.

The first result which was presented in this paper is the algorithm for the analyzing statuses of a single patient (OPTCAC). In this algorithm, any state of patient is compared with the state at the special time (mostly at the start of the therapy).

Algorithm GPTCAC analyzes results in two directions. First of these analysis are expectations of compared estimates and the second of these analysis are the compared expectations of estimates.

Algorithm CTCAC compares effects of many therapies applied on different groups of patients, in general. However, this algorithm may compare different effects of different doses of the same therapy.

The estimates of states of patient(s) are $\sigma$-additive and pseudo-additive measures. The curves which connect these estimates have the same measure-characteristics as the estimates which they connect.

This methodology estimates the separate systems of human beings but it estimates the complete states. It is the preferment of standard methodologies which may analyze just similar results.

3.1. Possible implementations. Five groups of medical tests were defined in this paper. There are some objective medical results which estimates are as same as the estimates of subjective tests. For this reason, the groups PNT and PPT are better classifications of these tests than the classification in subjective and objective tests (see [17]). Purpose 1 is completed in this way.

All estimates of results in tests from the group PPT are equalized as: 0 is the estimate of any negative result, 0.5 is the estimate of any the least intensive positive result and 1 is the estimate of any the most intensive positive result. This fact equalizes differences between different medical scales. Purpose 2 is completed in this way. 
Functional connections of estimates makes possible analysis of results in tests realized at different times. In this way, it is completed the Purpose 3.

Example 3.1: A patient with diabetic ketoacidosis is hospitalized in coma-state. This patient is primary ill of multiple sclerosis (MS) and secondary of diabetes mellitus. The state of coma is estimated with 15 by GCS [18] (all possible estimates are $3,4, \ldots, 15$ and it is the case mipr from the equation (2.3)). The state of multiple sclerosis of this patient is estimated with 6.5 by EDSS [7] (possible estimates of positive states are $1,1.5,2,2.5, \ldots, 10)$. It is found that sugar level before meal in blood of this patient was $157 \mathrm{mg} / \mathrm{dl}$ (the reference range is $70-99 \mathrm{mg} / \mathrm{dl}$ ).

After two days, patient was not in coma. The state of MS was estimated as 6.5 by EDSS and sugar level before meal in his blood is $128 \mathrm{mg} / \mathrm{dl}$.

Estimations of results: The state of coma be $\mathbf{r}_{1}^{2}$, the state of multiple sclerosis be $\mathbf{r}_{2}^{2}$ and the sugar level before meal in the patient's blood is $\mathbf{r}_{1}^{4}$. As we may see, the states of coma and multiple sclerosis are tests from the group PPT and the sugar levels before meal are tests from the group RRVT.

Application of OPTCAC:

The estimates of these results are

\begin{tabular}{|l|c|c|}
\hline & the first control & the second control \\
\hline \hline states of comma & 0.5 & 0 \\
\hline states of MS & 0.806 & 0.806 \\
\hline \hline sugar level & 2 & 1 \\
\hline
\end{tabular}

Based on the times $t_{1}=0$ days and $t=2$ days, there are not critical times.

Coma, in our case, is an acute disorder. MS and diabetes are acute diseases. We have $\left(3 t_{2}-t_{1}\right) / 2=3$. The coordinate matrix curves which connect the estimates of results are:

$$
\begin{array}{ll}
\text { coma states: } & m_{1}^{2}(t)=0.0625 t^{2}(t-3)+0.5 \\
\text { MS states: } & m_{2}^{2}(t)=0.806 \\
\text { sugar levels: } & m_{4}^{1}(t)=-0.25 t^{2}+2
\end{array}
$$

The corresponding TACs and CACs are

$$
\begin{array}{lll} 
& \text { TAC } & \text { CAC } \\
\text { coma states: } & \tau_{1}^{2}(t)=\left[0.125 t^{2}(t-3)+1\right] & c_{1}^{2}(t)=\left[0.03125 t^{3}-0.125 t^{2}+1\right], \\
\text { MS states: } & \tau_{2}^{2}(t)=[1] & c_{2}^{2}(t)=[1], \\
\text { sugar levels: } & \tau_{1}^{4}(t)=\left[-0.125 t^{2}+1\right] & c_{1}^{4}(t)=\left[-0.042 t^{2}+1\right] .
\end{array}
$$

The TACs and CACs of the neurological state (comma and MS) and the sugar level are $\tau_{1,2}^{2}(t)$ and $c_{1,2}^{2}(t)$ as well as $\tau_{1}^{4}(t)$ and $c_{1}^{4}(t)$, i.e.

$$
\tau_{1,2}^{2}(t)=\frac{1}{0.5+0.806}\left[m_{1}^{2}(t)+m_{2}^{2}(t)\right]=\left[0.048 t^{3}-0.144 t^{2}+1\right],
$$




$$
\tau_{1}^{4}(t)=\left[-0.125 t^{2}+1\right],
$$

such as

$$
\begin{aligned}
& c_{1,2}^{2}(t)=\left[0.120 t^{3}-0.048 t^{2}+1\right], \\
& c_{1}^{4}(t)=\left[-0.042 t^{2}+1\right] .
\end{aligned}
$$

The TAC and CAC whcih connect the complete statuses of this patient are

$$
\begin{aligned}
\tau(t) & =\left[\sqrt{0.001 t^{6}-0.004 t^{5}+0.017 t^{4}+0.029 t^{3}-0.261 t^{2}+1}\right], \\
c(t) & =\left[\sqrt{0.00004 t^{6}-0.00034 t^{5}+00.0019 t^{4}+0.00715 t^{3}-0.08704 t^{2}+1}\right] .
\end{aligned}
$$

Graphically and numerically, the changing of these estimates trough time is shown on Figure 3:
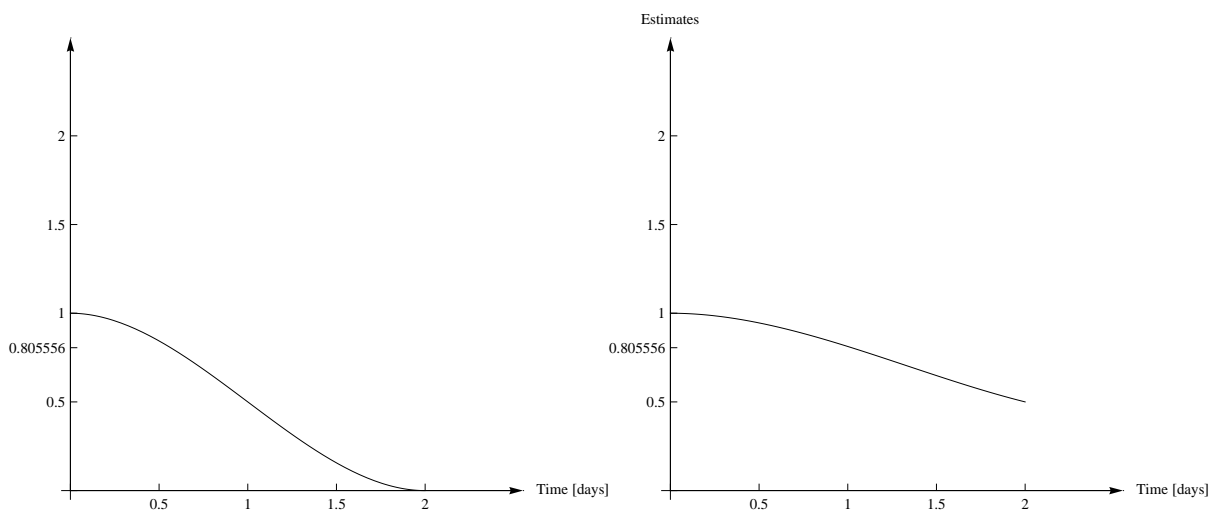

Figure 3. TAC (left) and CAC (right) of coma

From this figure, we see that the status of coma was in constant preferment. This status was estimated with 2 at the time $t=0$ and with 1 at $t=2$. This status was changed for 1 , i.e. for $1-1 / 2=0.5$. This change is for $50 \%$. Moreover, the state a patient becomes more and more stable trough time (see Figure 3).

This figure shows us that status of multiple sclerosis stays constant (estimated with 0.806 at $t=0$ and $t=2$ ). It is logical because for preferment of status in any chronic disease, it is necessary more than two days.

From the Figure 5, we see that complete neurological status is in preferment and became more stable trough these two days. Moreover, the estimate of neurologic state at the time $t=0$ was $m_{1}^{2}(0)+m_{2}^{2}(0)=1.306$. The estimate of neurologic status at the time $t=2$ is $m_{1}^{2}(2)+m_{2}^{2}(2)=1.056$. The initial status was preferred for $|1.306-1.056|=0.25$. In percentage, this preferment is $1-1.056 / 1.306=0.1915$, i.e. $19.15 \%$. 

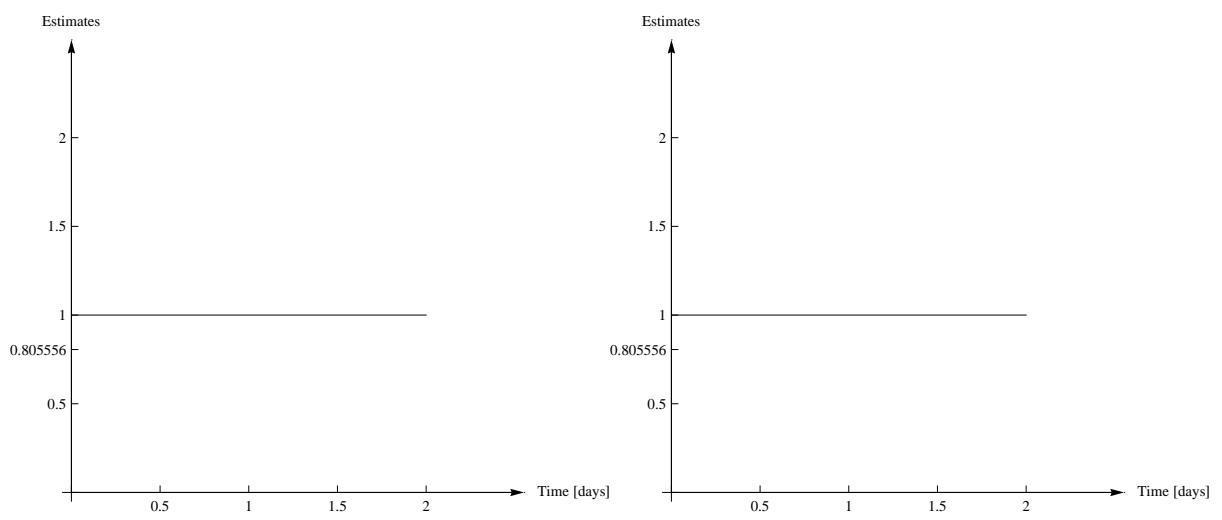

Figure 4. TAC (left) and CAC (right) of MS
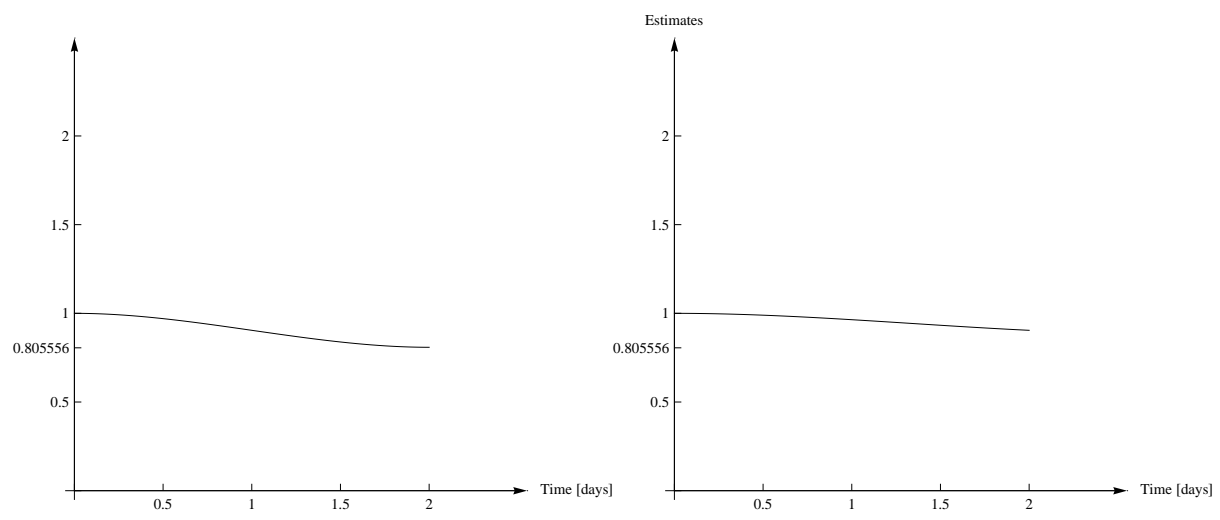

Figure 5. TAC (left) and CAC (right) of complete neurological state

We see that sugar levels are in constant preferment trough time (see Figure 6). At the time $t=0$, the status is estimated with 2 and with 1 at the time $t=2$. The difference between estimates is 1 . This change is for $1-1 / 2=0.5$, i.e. $50 \%$.

Complete status of this patient is preferred after two days (see Figure 7). Numerically, the status is estimated with 2.388 at the time $t=0$ and 1.454 at the time $t=2$. The preferment is for 0.934 , i.e. for $1-1.454 / 2.388=0.6088$, i.e. $60.88 \%$.

Remark 6: Unlike statistics, which results are probabilities that there is a difference between two groups of results, results of the applied method (as we may see) are how much the studied results change. It is more precise than any probability. 

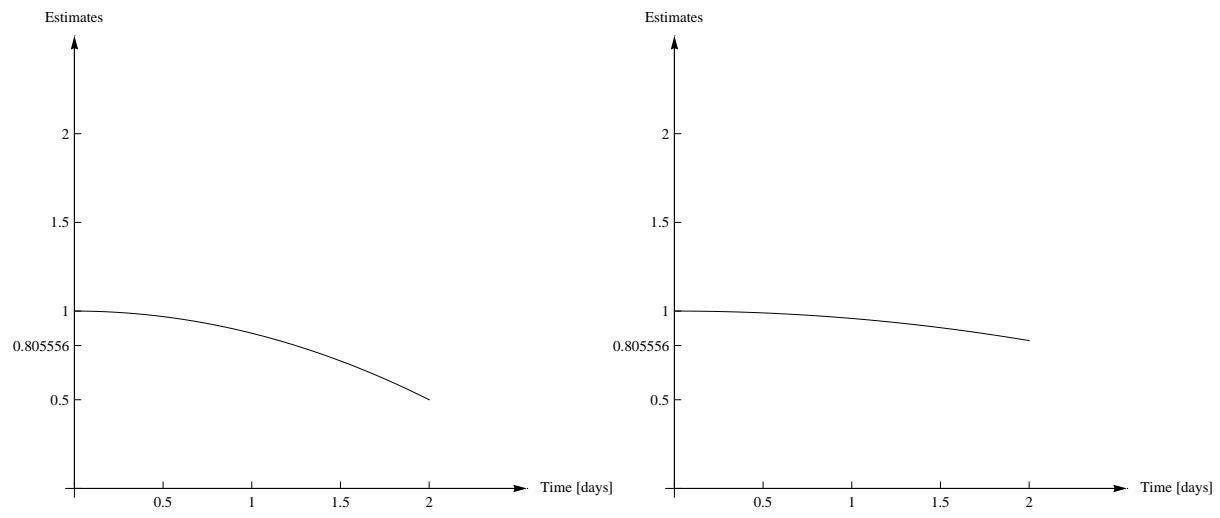

Figure 6. TAC (left) and CAC (right) of sugar levels
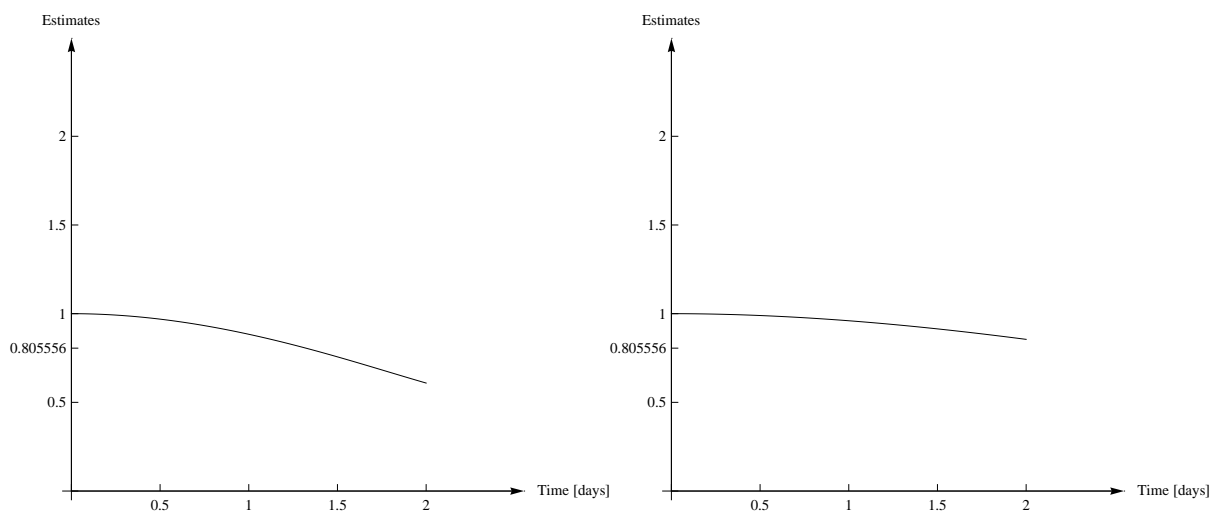

Figure 7. TAC (left) and CAC (right) of complete status

\section{REFERENCES}

[1] D. Bowers, Medical Statistics from Scratch: An Introduction for Health Professionals, 2nd edition,Wiley-Interscience,2008.

[2] F. M. Dekking, C. Kraaikamp, H. P. Lopuhaä, L. E. Meester, A Modern Introduction to Probability Statistics, Understending Why and How, Springer-Verlag, London, Limited, 2005.

[3] H. Federer, Colloquium Lectures on Geometric Measure Theory, BULLETIN OF THE AMERICAN MATHEMATICAL SOCIETY, 84 (3) (1978), pp. 291-338.

[4] K. Flaister, The presence of mathematics and computer anxiety in nursing students and their effects on medication dosage calculations, Nurse Education Today, 27 (2007), pp. 291-338.

[5] I. Jovanović, V. Rakočević, Multipliers of mixed-norm sequence spaces and measures of noncompactness, Mat. Vesnik, 49 (1997), 197-206.

[6] Ž. Krsmanović, M. Živković, T. Lepić, A. Stanković, R. Raičević, E. Dinčić, Small internal jugular veins with restricted outflow are associated with severe multiple sclerosis: a sonographer-blinded, case-control ultrasound study, BMC Neurology, 13:90, 2013.

[7] J. F. Kurtzke, Rating neurologic impairment in multiple sclerosis: an expanded disability status scale (EDSS), Neurology, 33 (11), (1983), pp. 1444-1452. 
[8] B. de Malafosse, V. Rakočević, Applications of measures of noncompactness of linear operators on the spaces, Journal of Mathematical Analysis and Applications, 323 (1), (2006), pp. 131-145.

[9] E. Malkowski, V. Rakočević, The measure of noncompactness of linear operators between certain sequence spaces, Acta Sci. Math. (Szeged), 64 (1998), pp. 151-170.

[10] S. Minčić, Lj. Velimirović, Differential Geometry of Curves and Surfaces, in Serbian, Faculty of Science and Mathematics, Niš, 2007.

[11] B. M. Mirković, Measure and Integral Theory, in Serbian, Naučna Knjiga, Belgrade, 1990.

[12] V. Rakočević, Measures of non-strict-singularity of operators, Mat. Vesnik, 35 (1) (1983), pp. 79-82.

[13] V. Rakočević, Measures of noncompactness and some applications, FILOMAT, 12:2 (1998), $87-120$.

[14] T.Shifrin, Differential Geometry: A First Course in Curves and Surfaces, Preliminary Version, Fall, 2014.

[15] M. Sugeno, T. Murofushi, Pseudo-additive Measures and Integrals, Journal of Mathematical Analysis and Applications, 122 (1) (1987), pp. 197-222.

[16] N. Vesić, Differential Geometry and Matrices, XVII Geometrical Seminar, Zlatibor 2012, available in:

http://poincare.matf.bg.ac.rs/ geometricalseminar/presentations/vesic.pdf.

[17] N. Vesić, D. Ilić, Testing Effects of a Drug: Drugs for Chronic Diseases, Proc of the ICTIC 2012, pp. 111-119.

[18] E. F. Wijdicks, W. R. Bamlet, B. V. Maramattom, E. M. Manno, R. L. McClelland, Validation of a New Coma Scale: The FOUR Score, Ann. Neurol., 58, 2005, pp. 585-593.

Nenad O. Vesić

UNiversity OF Niš,

Faculty of Science and Mathematics,

VišEgRADSKa 33,18000 Niš,

SERBia

E-mail address: vesko1985@pmf.ni.ac.rs

dr Lana Maćukanović-Golubović

UNIVERSITY OF Niš,

Medical Faculty,

Bulevar dr Zorana ĐinĐića 81, 18000 Niš,

Serbia

E-mail address: doktorlanagolubovic@gmail.com

DR Dus̃ica ILIĆ

UNIVERSITY OF Niš,

Medical Faculty,

Bulevar dr Zorana ĐinĐića 81, 18000 Niš,

Serbia

E-mail address: dr.dusica.ilic@gmail.com 